\title{
Nutritional Status and Asymptomatic Infection in Young Children in Two Different Endemic Areas after Seasonal Malaria Chemoprevention Campaign in Mali
}

\author{
Drissa Konaté ${ }^{1 *}$, Moctar Tounkara ${ }^{2}$, Sory I Diawara ${ }^{1}$, Ibrahim M Sissoko ${ }^{1}$, Larissa Denou ${ }^{1}$, \\ Bourama Keita $^{1}$, Fatoumata Kassé ${ }^{1}$, Oumar Diarra ${ }^{1}$, Nafomon Sogoba ${ }^{1}$, Mahamadou Diakité ${ }^{1}$ \\ and Seydou Doumbia ${ }^{1}$ \\ ${ }^{1}$ West African International Center for Excellence in Malaria Research (ICEMR-WA), University of Sciences, Techniques and Technologies of Bamako, Mali \\ ${ }^{2}$ Department of Education and Research in Public Health and Specialties (DERSP), University of Sciences, Techniques and Technologies of Bamako, Mali
}

*Corresponding author: Drissa Konaté, West African International Center for Excellence in Malaria Research (ICEMR-WA); Faculties of Medicine and Odontostomatology, and Pharmacy; University of Sciences, Techniques and Technologies of Bamako, Bamako, Mali.

To Cite This Article: Drissa Konaté, Moctar Tounkara, Sory I Diawara, Ibrahim M Sissoko, Larissa Denou, et al., Nutritional Status and Asymptomatic Infection in Young Children in Two Different Endemic Areas after Seasonal Malaria Chemoprevention Campaign in Mali. Am J Biomed Sci \& Res. 2021 - 12(6). AJBSR.MS.ID.001801. DOI: 10.34297/AJBSR.2021.12.001801.

Received: 㘹 April 26, 2021; Published: 筒 May 07, 2021

\begin{abstract}
Undernutrition represents an additional burden in Mali where malaria remains a major public health problem. A cross sectional survey was carried out in November 2018 and 2019 at the end of the Seasonal Malaria Chemoprevention (SMC) campaign to investigate the association between asymptomatic infection and nutritional status. A total of 541 children aged 6 to 59 months were included, 233 (43.0\%) in Koila Bamanan (in an irrigated area with longer seasonal malaria transmission) and 308 (57.0\%) in Kita. Data on parasitemia and nutritional status were collected and analyzed using logistic regression. Overall, underweight prevalence was $16.9 \%$ in Kita vs. $14.6 \%$ in Koila ( $p=0.4$ ); $25.6 \%$ vs. $40.8 \%$ (p $=0.001$ ) for stunting and $14.9 \%$ vs. $10.7 \%(\mathrm{p}=0.001)$ for wasting in Kita and Koila, respectively. The prevalence of asymptomatic infection was $17.9 \%$ in Kita vs. $6.2 \%$ in Koila among children $6-23$ months $(\mathrm{p}=0.045)$. In multivariate analysis, anemia was associated with underweight (adjusted $\mathrm{OR}=2.92$; 95\%IC [1.59-5.38]), and stunting (adjusted OR $=3.17$, 95\%IC [2.02-4.98]). Asymptomatic infection was associated with underweight (adjusted $\mathrm{OR}=2.41,95 \% \mathrm{IC}$ [1.21-4.80]. Stunting was higher in children living in Koila (adjusted OR $=2.44,95 \% \mathrm{IC}$ [1.60-3.71]. However, the reduction in stunting was observed in children 24-59 months (adjusted OR $=0.4495 \% \mathrm{CI}$ [0.28-0.96]). A significant correlation was observed between parasite density and underweight in Kita (0.049). Undernutrition remains high among children in both areas and was associated with asymptomatic malaria infection.
\end{abstract}

Keywords: Nutritional status, Asymptomatic infection, Young children, Mali

Abbreviations: ACF/AAH: Action Contre la Faim/Action Against Hunger; ENA: Emergency Nutrition Assessment; GIS/UCRC: Geographic Information System/ University Clinical Research Center; ICEMR: International Center for Excellence in Malaria Research; MPV: Millennium Promise Village; NGO: Non-Governmental Organization; NMCP: National Malaria Control Program; SMART: Standardized Monitoring and Assessment of Relief and Transitions; WHO: World Health Organization

\section{Background}

Undernutrition represents an additional burden in Sub-Saharan Africa countries where malaria remains a major public health problem [1]. Most of deaths in children under five years linked to undernutrition occur in low-income countries [2]. National

prevalence of undernutrition varies according to residence areas in Mali. The various forms of undernutrition represent respectively $24.1 \%, 18.6 \%$ and $10 \%$ for stunting, underweight and wasting in children under five years [3]. 
Among factors associated to undernutrition occurrence in children under five years, infectious disease, especially malaria and geohelminth were mainly reported in sub-Saharan Africa countries [4-6]. Undernutrition and malaria are common in children under 5 years old, mutually worsen each other especially in malaria endemic areas. Their coexistence has a negative impact on child's learning, cognitive and physical development and responsible of most death in target population $[5,7,8]$.

World Health of Organization (WHO) recommends Seasonal Malaria Chemoprevention (SMC) to prevent malaria in children under five years during malaria transmission in the sub-Saharan Africa region. It consists to monthly treatment of SulfadoxinePyrimethamine (SP) plus Amodiaquine (AQ) during 4 months in high transmission season. Children received SP the 1st day and AQ for 3 days [9]. The authors reported that SMC has effective, safe and cost-effective to prevent malaria in target population [10-12] and could improve children's nutritional status in malaria endemic areas during it implementation period. This study was carried out in two endemic areas: Koila village, endemic malaria area Northeast from Bamako and Kita, another endemic area West from Bamako; to investigate the association between nutritional status and malaria after the last round of SMC and the possible effect of SMC in nutritional status in perspective to combine the two diseases control interventions ongoing.

\section{Materials and Methods}

\section{Study Site and Participants}

This study is part of pilot study on SMC implementation in Kita funded by Save the Children and President's Malaria Initiative (PMI), and International Center for Excellence in Malaria Research (ICEMR) on malaria control interventions impact in Koila, both in collaboration with Malian National Malaria Control Program (NMCP). The study was carried out in two eco-climatic areas:

a) Koila Village, at $45 \mathrm{~km}$ from Markala district health and $385 \mathrm{~km}$ Northeast of Bamako. It is an irrigated area with longer seasonal malaria transmission. Malaria control interventions ongoing are seasonal malaria chemoprevention, malaria cases management after rapid test diagnosis, use of insecticide treated nets and intermittent preventive treatment in pregnant women. Koila was a millennium challenge experimental site until 2016 and currently ICEMR project, UNICEF and Terre des Hommes International non-governmental organization doing research on malaria and malnutrition, respectively.

b) Kita, another endemic area at $186 \mathrm{~km}$ West of Bamako with many rivers. Malaria transmission is also intense during rainy season. President's Malaria Initiative (PMI) and Action Against Hunger has undertaken different activities in children since 2014 in collaboration with NMCP.

\section{Study Design \& Data Collection}

The cross-sectional survey was carried out in November 2018 in Kita and 2019 in Koila, one month after the last round of SMC campaign to investigate association of children's nutritional status and malaria infection. The study protocol has already been approved by Ethical Committee of Faculties of Medicine, Pharmacy and Odontostomatology of University of Sciences, Techniques and Technologies of Bamako. After obtaining written inform consent from the parents/guardians, temperature, age, weight and height were measured in each child during physical examination, blood sample were collected to perform blood smear and measure hemoglobin level.

Blood smear was used to determine parasitemia by microscopy. The thick blood films were stained with $10 \%$ Giemsa and examined under the $\times 100$ oil immersion objective lens of a light microscope. The number of asexual parasites was counted on 200 leucocytes and sexual parasites on 1000 leucocytes. Hemoglobin level was measured from whole blood using Hemocue 301. Electronic thermometer was used to determine axillary temperature. Malaria infection was defined as having a positive blood smear with Plasmodium, anemia by Hemoglobin $(\mathrm{Hb})<11 \mathrm{~g} / \mathrm{dl}$ and the fever for any temperature $>37.5^{\circ} \mathrm{C}$. Each case of malaria or undernutrition has been referred to community health center for best management.

\section{Sample Calculation \& Data Analysis}

Sample size has been estimated from the national prevalence of undernutrition among children under five years (23.1\%) in Mali, assuming a confidence level at 95\%, cluster effect at 2 and $10 \%$ of lost to follow up, a sample size was 542 participants. Data on parasitemia and nutrition were collected by using electronic data capture and exported in Excel 2010 and analyzed with STATA 14. Anthropometric indices were calculated with ENA for SMART. Logistic regression was used to determine association between nutritional status of children and malaria infection. Pearson correlation was used to determine the correlation between parasite density and z-score of anthropometric indices with a significant $\mathrm{p}$ value at 0.05 .

Asymptomatic malaria infection was defined as having Plasmodium falciparum in blood smear without fever, and anemia was defined as Hemoglobin $(\mathrm{Hb})<11 \mathrm{~g} / \mathrm{dl}$. The undernutrition was defined by z-score < -2 standard deviation for height/age (stunting), weight/age (underweight) and weight/height (wasting) [13].

\section{Results}

A total of 541 children under five years were enrolled in this study. Age group 24-59 months represents $81.8 \%$ in Kita vs. $72.1 \%$ in Koila, and $18.2 \%$ vs. $27.9 \%$ for those $6-23$ months. The prevalence of asymptomatic malaria infection was $17.9 \%$ vs. $6.2 \%$ among 
children 6-23 months in Kita compared to Koila ( $\mathrm{p}=0.045) ; 15.5 \%$ vs. $14.9 \%$ for those $24-59$ months $(\mathrm{p}=0.8)$. Anemia prevalence was $63.9 \%$ among $24-59$ months in Kita against $48.2 \%$ in Koila (p $=0.001$ ); and similar between those 6-23 months in both areas. Underweight prevalence was $23.2 \%$ vs. $18.5 \%$ among $6-23$ months in Kita compared to Koila ( $\mathrm{p}=0.5$ ); and 15.5 vs. $13.1 \%$ in $24-59$

Table 1: Characteristics of participants in Kita and Koila. months ( $\mathrm{p}=0.4)$. Among children 6-23 months, stunting was high in Koila (73.9\%) compared to Kita (19.6\%) ( $p=0.001)$; and similar in those 24-59 months. The prevalence of wasting was $28.6 \%$ in Kita vs. $1.5 \%$ in Koila among children 6-23 months ( $\mathrm{p}=0.001)$; but similar in 24-59 months (Table 1).

\begin{tabular}{|c|c|c|c|c|c|c|}
\hline \multirow{2}{*}{ Characteristics } & \multicolumn{3}{|c|}{$6-23$ months } & \multicolumn{3}{c|}{ 24-59 months } \\
\cline { 2 - 6 } & Kita n (\%) & Koila n (\%) & p & Kita n (\%) & Koila n (\%) & p \\
\hline Parasitemia & $10(17.9)$ & $4(6.2)$ & 0.045 & $39(15.5)$ & $25(14.9)$ & 0.8 \\
\hline Anemia & $39(69.6)$ & $42(64.6)$ & 0.5 & $161(63.9)$ & $81(48.2)$ & 0.001 \\
\hline Gametocyte carriage & $4(7.1)$ & $3(4.6)$ & 0.5 & $12(4.8)$ & $11(6.6)$ & 0.6 \\
\hline Underweight & $13(23.2)$ & $12(18.5)$ & 0.5 & $39(15.5)$ & $22(13.1)$ & 0.4 \\
\hline Stunting & $11(19.6)$ & $48(73.9)$ & 0.001 & $68(27)$ & $47(28)$ & 0.8 \\
\hline Wasting & $16(28.6)$ & $1(1.5)$ & 0.001 & $30(11.9)$ & $24(14.3)$ & 0.4 \\
\hline
\end{tabular}

For afebrile children, multivariate analysis revealed that strong risk to develop stunting (AOR $=2.44,95 \%$ CI [1.60-3.71]. anemia was associated with underweight $(\mathrm{AOR}=2.92 ; 95 \%$ However, age group 24-59 months was associated with stunting CI [1.59-5.38]) and stunting (AOR $=3.17,95 \%$ CI [2.02-4.98]). reduction in the both areas (AOR = 0.44 CI 95\% [0.28-0.96]). Any Asymptomatic malaria infection was associated with underweight significant association between wasting and parasitemia, anemia $($ AOR $=2.41,95 \%$ CI [1.21-4.80]). Children living in Koila had a and gametocyte carriage was found (Table 2).

Table 2: Associated factors with nutritional status among afebrile children in both sites.

\begin{tabular}{|c|c|c|c|c|c|c|c|c|c|}
\hline \multirow{2}{*}{$\begin{array}{c}\text { Independents } \\
\text { Variables }\end{array}$} & \multicolumn{3}{|c|}{ Underweight } & \multicolumn{3}{c|}{ Stunting } & \multicolumn{2}{c|}{ Wasting } \\
\cline { 2 - 10 } & AOR & CI 95\% & P & AOR & CI 95\% & P & AOR & CI 95\% & P \\
\hline Study site & & & & & & & & \\
\hline Kita & Ref. & & & Ref. & & & Ref. & \\
\hline Koila & 0.9 & {$[0.53-1.53]$} & 0.7 & 2.44 & {$[1.60-3.71]$} & 0.001 & 0.75 & {$[0.42-1.33]$} & 0.3 \\
\hline Age group & & & & & & & & & \\
\hline 6-23 months & Ref. & & & Ref. & & & Ref. & & \\
\hline 24-59 months & 0.66 & {$[0.38-1.17]$} & 0.1 & 0.44 & {$[0.28-0.96]$} & 0.001 & 0.79 & {$[0.42-1.48]$} & 0.4 \\
\hline $\begin{array}{c}\text { Asymptomatic } \\
\text { parasitemia }\end{array}$ & 2.41 & {$[1.21-4.80]$} & 0.01 & 1.43 & {$[0.75-2.71]$} & 0.2 & 1.88 & {$[0.87-4.03]$} & 0.1 \\
\hline Gametocyte carriage & 0.41 & {$[0.12-1.36]$} & 0.1 & 0.56 & {$[0.21-1.45]$} & 0.2 & 0.53 & {$[0.14-2.01]$} & 0.3 \\
\hline Anemia & 2.92 & {$[1.59-5.38]$} & 0.001 & 3.17 & {$[2.02-4.98]$} & 0.001 & 1.02 & {$[0.58-1.80]$} & 0.9 \\
\hline
\end{tabular}

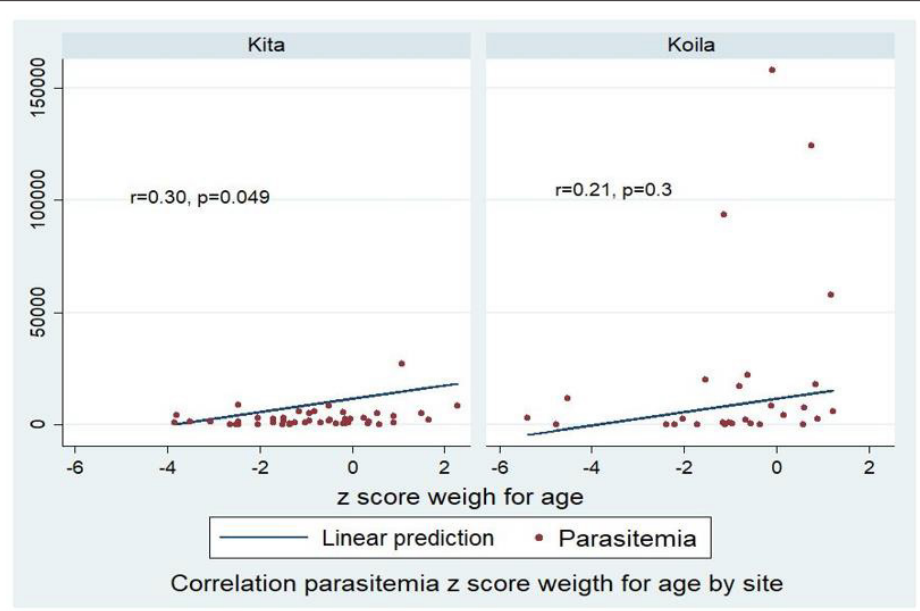

Figure 1: Correlation between parasite density and anthropometric indices weight for age. 
A significant correlation was found between parasite density and $z$-score of underweight children in Kita ( $r=0.30, p=0.049$ ) (Figure 1). This correlation was not significant in wasting children
(Figure 2), but a trend of small decrease in parasite density in children having stunting in both study sites was observed (Figure $3)$.

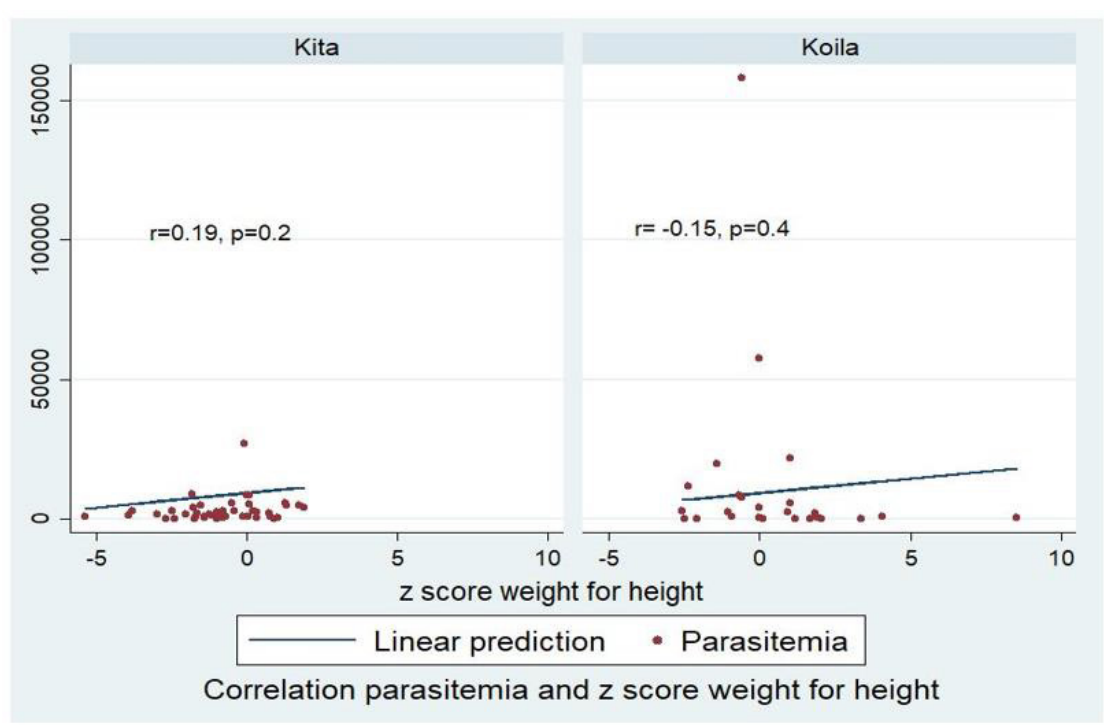

Figure 2: Correlation between parasite density and anthropometric indices weight of height.

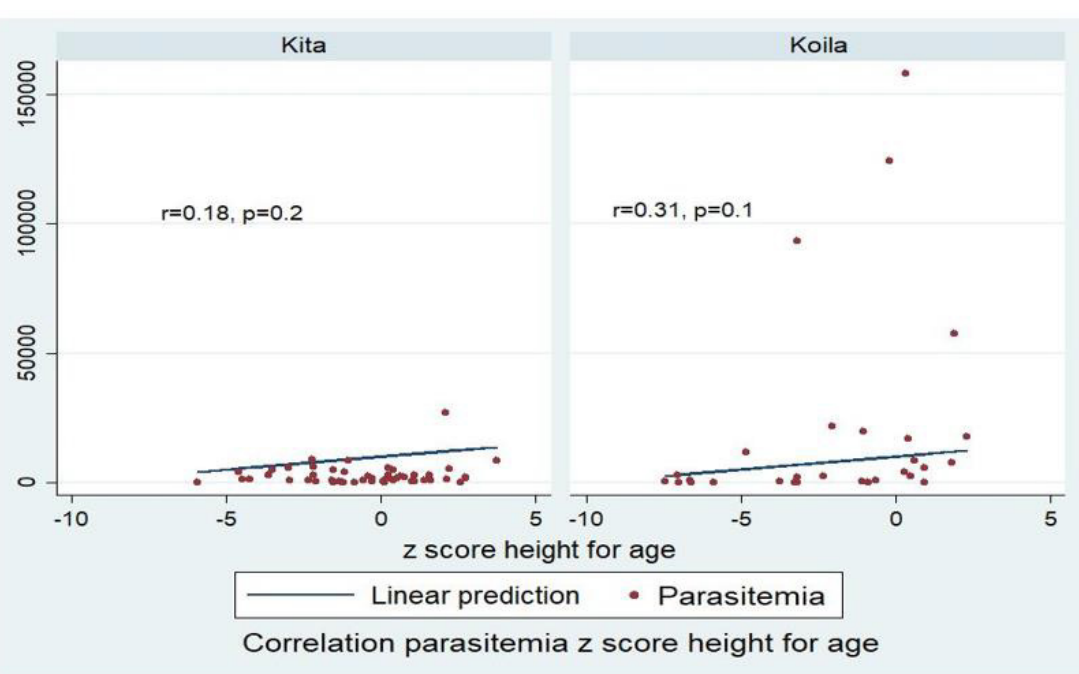

Figure 3: Correlation between parasite density and anthropometric indices height for age.

\section{Discussion}

Malaria and undernutrition are frequently associated Malian children under five years and are a major cause of their mortality [14]. This study was carried out to investigate undernutrition and their association with malaria infection among children living in two endemic areas after SMC campaign. Children aged 24 to 59 months were the most represented in both sites (Table 1) as reported generally in Mali $[3,15]$. Malaria infection was frequent among children aged 6 to 23 months in Kita ( $p=0.045)$, but similar in those 24-59 months in both sites $(p=0.8)$. Otherwise, the prevalence of anemia was higher among 24-59 months in Kita $(\mathrm{p}=0.001)$. Anthropometric indices of children nutritional status varied according to residence areas and age groups. Underweight and wasting were more frequent in Kita and stunting in Koila among children 6-23 months, but it was similar among children aged 24 to 59 months in both sites (Table 1 ).

Undernutrition situation remains a major concern in terms of public health in both sites [3], despite the efforts of the NonGovernmental Organization (NGO) as Action Against Hunger (AAH) in Kita and Millennium Promise Village in Koila to ensure safe water, good health and food self-sufficiency. Since 2007, AAH was deployed in Kita District and conduct activities in different areas 
as health, nutrition, education and safe water. Millennium Promise Village from 2006 to 2016 worked on the same field in Koila, and currently Terre des Hommes carry out activities in Dioro area on food security.

Using logistic regression, anemia was associated with underweight (AOR $=2.92 ; 95 \% \mathrm{CI}$ [1.59-5.38]) and stunting (AOR $=3.17,95 \%$ CI [2.02-4.98]). An association was found between asymptomatic malaria infection and underweight $(\mathrm{AOR}=2.41$, 95\% CI [1.21-4.80]. The risk to develop stunting was higher among children living in Koila after adjusting on confounding factors (AOR $=2.44,95 \%$ CI [1.60-3.71]). However, a decrease in stunting was observed in children aged 24 to 59 months $(\mathrm{AOR}=0.44,95 \% \mathrm{CI}$ [0.28-0.96]) in the two sites. Any significant association was found between wasting and asymptomatic malaria infection, anemia and gametocyte carriage (Table 2). The role of infectious diseases, particularly Plasmodium falciparum infection in undernutrition occurrence among children has been already demonstrated in subAfrican malaria endemic areas [16,17].

Children living in a pathogenic environment where they are exposed to repeat infectious diseases contribute to nutrient deficit, leads food imbalance and thereafter undernutrition [7,14]. Also, a repetitive infectious disease quickly induces malnutrition among children already underweight in those areas [18-20]. A small significant correlation was found between parasite density and underweight in Kita ( $\mathrm{r}=0.3, \mathrm{p}=0.049)$ (Figure 1). Any significant correlation was not found between parasite density and others stunting and wasting according to anthropometric indices, but a small trend in parasite density decrease in children having stunting was observed in both sites (Figure 3). In Plasmodium falciparum endemic areas, children having stunting and underweight were more infected by Plasmodium, but parasite density remains lower $[21,22]$.

Seasonal malaria chemoprevention with SP/AQ decrease malaria infection and reduce Plasmodium falciparum parasite density in children. All children in this study received four rounds of SMC during malaria season. Combine nutritional interventions to seasonal malaria chemoprevention in malaria endemic areas could reduce parasite density and improve nutritional status in children [23]. Other parameters as family income, mother's characteristics, other pathologies were not investigated and could be influenced children nutritional status [24-26] are the limit of this study.

\section{Conclusion}

Undernutrition remains high among children in both sites and was associated with asymptomatic malaria infection. More studies are required to assess the treatment compliance during SMC and investigate another factor associated with nutritional status in children.

\section{Declaration}

Ethic approval and Consent to participate. This study as part of the ICEMR protocol was already approved by ethical committee of Faculty of Medicine, Pharmacy and Odontostomatology, University of Sciences, Techniques and technology of Bamako under $\mathrm{N}^{0} 2019 / 04 / \mathrm{FMPOS}$ revised. All participants given their agreement before data collection.

\section{Consent for Publication}

i. All authors read and approved the final version of the manuscript before submission.

ii. Availability of data and materials

iii. Contact the corresponding author for any information about data availability.

\section{Conflict of Interest}

The authors declare any competing interests.

\section{Funding}

This study as part of ICEMR project, sponsored by the National Institute of Allergy and Infectious Diseases (NIAID). Grants 1-U19 AI 129387-01 WEST AFRICA ICEMR AWARD. Protocol DMID Number: 17-0052. But for this study include to ICEMR program any funding was not found.

\section{Author's contributions}

MD, SD, SD: Study design

LD and BK: data acquisition

DK, SD and MT: Data analysis and interpretation

DK, SD and MT: wrote main manuscript text.

DK, SD, MT, IS, NS, FK: reviewed the manuscript.

Final version: MD, SD

\section{Acknowledgements}

ICEMR project, National Malaria Control Program of Mali, Communities of Kita and Koila, all participants and investigators, Jules Mihigo and Celia Jane Woodfill from PMI to support SMC implementation in Kita.

\section{References}

1. Nubé M, Sonneveld BGJS (2005) The geographical distribution of underweight children in Africa. The International Journal of Public Health 83(10): 764-770.

2. OMS Malnutrition (2020)

3. DNS/DN/INSTAT (2018) Inquiry Nationale Nutritionnelle and Mortalit Ritrospective suivant la mithodology SMART, Mali. pp.1-119. 
4. Adebami OJ, Owa JA, Oyedeji GA, Oyelami OA (2007) Prevalence and problems of fetal malnutrition in term babies at Wesley Guild Hospital, South Western Nigeria. West Afr J Med 26(4): 278-282.

5. Fillol F, Cournil A, Boulanger D, Cissé B, Targett G, et al. (2009) Influence of wasting and stunting at the onset of the rainy season on subsequent malaria morbidity among rural preschool children in Senegal. Am J Trop Med Hyg 80(2): 202-208.

6. Krawinkel MB (2012) Interaction of nutrition and infections globally: an overview. Ann Nutr Metab 61(1): 39-45.

7. Bernard OH, Pagezy Bley D (2001) Nutritiontat nutritionnel and environnement pathogens d'enfants d'une population foresters du Sud-Cameroun 13: 1-2.

8. Christine C (2008) Inquire on llimentation des personas searches 396 : 44-45.

9. WHO (2012) World Malaria Report pp.195.

10. Ambe JP, Balogun ST, Waziri MB, Nglass IN, Saddiq A (2020) Impacts of Seasonal Malaria Chemoprevention on Malaria Burden among under Five-Year-Old Children in Borno State, Nigeria. Journal of Tropical Medicine 2020: 9372457.

11. Diawara F, Steinhardt LC, Mahamar A, Traore T, Kone DT, et al. (2017) Measuring the impact of seasonal malaria chemoprevention as part of routine malaria control in Kita, Mali. Malar J 16(1): 325.

12. Konate D, Diawara SI, Touré M, Diakité SAS, Guindo A, et al. (2020) Effect of routine seasonal malaria chemoprevention on malaria trends in children under 5 years in Dangassa, Mali. Malar J 19(1): 137.

13. WHO (2010) Nutrition Landscape Information System (NLIS). Country profile indicators Interpretation Guide p.51.

14. Gone T, Fiseha L, Eliso E, Samuel Y, Yohannes T (2017) The association between malaria and malnutrition among under-five children in Shashogo District, Southern Ethiopia: a case-control study. Infect Dis Poverty 6(1): 9.

15. EDS (2018) Inquire Dimographique et de Santé. Manuel du chef d'équipe et de la contrôleuse pp.1-52.

16. Tanner M, Burnier E, Mayombana C, Betschart B (1987) Longitudina study on the health status of children in a rural Tanzanian community: parasitoses and nutrition following control measures against intestinal parasites. Acta Trop 44(2): 137-174.

17. Wilson WM, Dufour DL, Staten LK, Barac Nieto M, Reina JC, et al. (1999) Gastrointestinal parasitic infection, anthropometrics, nutritional status, and physical work capacity in Colombian boys 11(6): 763-771.

18. Akiyama T, Pongvongsa T, Phrommala S, Taniguchi T, Inamine $\mathrm{Y}$, et al (2016) Asymptomatic malaria, growth status, and anaemia among children in Lao People's Democratic Republic: a cross-sectional study. Malar J 15(1): 499

19. Musimwa AM, Kanteng GW, Kitoko HT, Luboya ON (2016) Clinical signs of malnutrition in children living around a mining area: a case study in the city of Lubumbashi and its surrounding. Pan Afr Med J 24: 67.

20. Sumbele IU, Bopda OSM, Kimbi HK, Ning TR, Nkuo Akenji T (2015) Nutritional status of children in a malaria meso endemic area: cross sectional study on prevalence, intensity, predictors, influence on malaria parasitaemia and anaemia severity. BMC Public Health 15: 1099.

21. Ahmad SH, Moonis R, Shahab T, Khan HM, Jilani T (1985) Effect of nutritional status on total parasite count in malaria. The Indian Journal of Pediatrics 52(3): 285-287.

22. Saka AO, Saka MI, Adeboye Mohammed AN, Mokuolu Olugbenga A, Abu Saeed MB, et al. (2012) Malaria Parasitaemia in Children with Protein Energy Malnutrition in North Central Nigeria: A Case Control Study. IOSR Journal of Pharmacy and Biological Sciences 1(3): 49-53.

23. Ward A, Guillot A, Nepomnyashchiy LE, Graves JC, Maloney K, et al. (2019) Seasonal malaria chemoprevention packaged with malnutrition prevention in northern Nigeria: A pragmatic trial (SMAMP study) with nested case-control. PLOS ONE 14(1): 0210692.

24. Caroline MS, Leopold KF, Fatou D, Honafing D, Victor AS, et al. (2018) Predictors of poor nutritional status among children aged 6 and 24 months in agricultural regions of Mali: a cross-sectional study. BMC Nutrition 4(1): 18

25. Deribew A, Alemseged F, Tessema F, Sena L, Birhanu Z, et al. (2010) Malaria and under-nutrition: a community-based study among under-five children at risk of malaria, south-west Ethiopia. PLoS One 5(5): 10775.

26. Tine RC, Faye B, Ndour C, Sylla K, Sow D, et al. (2013) Parasitic Infections among Children under Five Years in Senegal: Prevalence and Effect on Anaemia and Nutritional Status. ISRN Parasitol pp.1-6. 